\title{
Design of Learning Resource Management Module in Personalized Intelligent Learning System
}

\author{
Changping Qi ${ }^{a}$, Youfang $\mathrm{Xu}^{\mathrm{b}}{ }^{\mathrm{w}}$ * \\ College of Information Technology and Media, Hexi University, Zhangye, Gansu, 734000 \\ a hxu102@163.com, ${ }^{\mathrm{b}}$ adele@hxu.edu.cn \\ *corresponding author: Jianjun $\mathrm{Wu}$
}

Keywords: personalized learning system; learning resource model; learning style model

\begin{abstract}
This paper firstly presents domestic and foreign research status of personalized learning system and then stresses that the most important personalities for learners are learning interest and learning style. On the basis of summarizing research status of personalized learning system, this paper offers a new design scheme. It refers to designing vector models and style models respectively for learning resources in accordance with text and non-text. At last, this paper elaborates modeling methods for these models.
\end{abstract}

\section{Introduction}

Personalized learning system, which develops on the pattern of Intelligent Tutoring System (ITS), has become one of significant study hotspots on intelligent education at present. Among all studies concerning personalized learning system, most of them were undertaken in universities and few research institutions at first. Domestically, Professor Yu Shengquan of Beijing Normal University conducts his study in the field of adaptive learning system; a team led by Professor Wang Lu of Capital Normal University studies the development of Personalized Courseware Generation System, as well as Professor Zhao Wei and Doctor Jiang Qiang of Northeast Normal University make a study of adaptive learning system. Foreign studies include InterBook (a study finding from an American called Brusilovsky), ELM-ART (a joint study made by a German named Weber and an American called Brusilovsky), INSPIRE (a Hypermedia System for personalized education designed and developed by Papanikolaou and other members at University of Athen)

As for learners' personalities, learning interest and learning style are two dominant features which can affect learners' learning efficiency. Most of existing studies are based on a learner's sole personality, while studies in which both learning interest and learning style are involved in the process of user modeling are fewer. Regarding studies of learning resources, some just realize the management of textual learning materials, that is, offering resources only and putting aside personalized recommendation; some achieve personalized recommendation to some degree, but they burden resource matching or get an imperfect matching result; Besides, some studies only implement text models in learning resources, but they do not consider multi-media learning resources, nor do they take into account the model matching with learners' learning style. Therefore, this paper offers a new scheme. It focuses on making respective models according to the two key personalities, namely, learners' learning interest and learning style; then discovering effective information through learners' learning behavior so as to timely update their interest models and learning style models; next, confirming learning style models in correspondence to learning resources on the basis of learners' learning style and their evaluation on resources recommended by the system; and finally, reasonably recommending learning resources to learners through scientific recommendation technology so as to realize personalized learning more efficiently.

\section{Design of Learning Resource Management Module}

For the sake of better matching learning resources, this paper offers diverse designs for learning 
resources at textual and non-textual level.

1) In terms of textual resources, this paper firstly carries out an array of pre-processing operations to textual resources such as Chinese word segmentation, word frequency statistics and synonymy combination. After these pre-processing operations, an $\mathrm{N}$-dimensional eigenvector model is created for every corresponding text. The textual vector space model is adopted to represent the eigenvector model so as to precisely express original information of texts as well as effectively reduce complexity of following calculation.

2)Considering non-textual resources, on the basis of key words and description of resources given by teachers, a corresponding $\mathrm{N}$-dimensional vector space model is shaped for this learning resource.

3)According to learners' learning style and their evaluation offered in the process of learning, a learning style model is made in correspondence to its learning resource.

Therefore, this paper centers on making two models for learning resources, namely, eigenvector models and learning style models. Eigenvector models can be divided into textual ones and nontextual ones.

\subsection{Construction of Textual Learning Resource Models}

In personalized learning system, we adopt a textual vector space model (VSM) to make an eigenvector model for textual learning resources. The method and procedures of making a textual learning resource model are as follows:

1)Word segmentation is reduced in all textual learning resources and frequency of all key words occurred is counted. The vector space model can be showed below:

$$
\text { Resource (document) }=\left\{\left(\mathrm{k}_{1}, \theta_{1}\right),\left(\mathrm{k}_{2}, \theta_{2}\right) \ldots\left(\mathrm{k}_{\mathrm{n}}, \theta_{\mathrm{n}}\right)\right\} \quad 1 \leq \mathrm{i} \leq \mathrm{n}
$$

Among that, “document” refers to “文档”; “ $k_{i}$ ” represents the ith key word in the document, and " $\theta_{\mathrm{i}}$ " stands for frequency of ith key word occurred in the document.

2)Since a title of textual resource can directly reflect crucial information of a document, the system will judge all key words in the title of the document. If the title of this document contains " $m$ " key words, then $k_{i}(0 \leq i \leq m)$ will be examined. If $k_{i}$ appears in the textual eigenvector model "Resource (document)", then the third procedure will follow; if not, the fourth procedure will be the next one.

3)If $k_{i}$ appears in the textual eigenvector model "Resource (document)" and takes the ith place of all vectors, then make $\theta_{\mathrm{i}}=\theta_{\mathrm{i}}+3 \times \mathrm{c}$. "c" stands for the proportion of times the key word occurred in the title of the document and the number of words of the document.

4)If $k_{i}$ doesn't occur in the textual eigenvector model "Resource (document)", the dimension number of the vector " $n=n+1$ " will be added. New Resource (document) will be as follows:

$$
\text { Resource (document) }=\left\{\left(\mathrm{k}_{1}, \theta_{1}\right),\left(\mathrm{k}_{2}, \theta_{2}\right) \ldots\left(\mathrm{k}_{\mathrm{n}+1}, \theta_{\mathrm{n}+1}\right)\right\}
$$

Among that, $k_{n+1}=k_{i}, \theta_{n+1}=3 \times c$. "c" represents the proportion of times the key word occurred in the title of the document and the number of words of the document.

5)Normalization will go on for all key words. That is,

$$
\theta_{i}=\frac{\theta_{i} \times \log \left(\frac{M}{N_{i}}+0.01\right)}{\sqrt{\sum \theta_{i}^{2} \times \log \left(\frac{M}{N_{i}}+0.01\right)^{2}}}
$$

" $M$ " refers to the total number of documents, and $N_{i}$ represents the number of documents which the ith key word appears. In order to avoid getting considerable eigenvector models and increasing complexity of following calculation, all textual learning resources are specified in descending order of their importance. And the first 20 key words will be taken as the final eigenvector models for learning resources.

The eigenvector model for each piece of learning resource is generated automatically by the 
system when learning resource is uploaded by teachers. It is stored in the knowledge base together with learning resource.

\subsection{Construction of Non-textual Learning Resource Models}

For non-textual learning resource, the vector space model (VSM) is also used to make an eigenvector model. The method and procedures of making a non-textual learning resource model are similar to those of making a textual learning resource model. The difference lies in that the former relies on description of resources, which is uploaded simultaneously with learning resources by teachers, to make a model. The procedures are below:

1)Word segmentation is reduced in all non-textual learning resources and frequency of all key words occurred is counted. The vector space model is as follows:

$$
\text { Resource }(\text { media })=\left\{\left(\mathrm{k}_{1}, \theta_{1}\right),\left(\mathrm{k}_{2}, \theta_{2}\right) \ldots\left(\mathrm{k}_{\mathrm{n}}, \theta_{\mathrm{n}}\right)\right\} \quad 1 \leq \mathrm{i} \leq \mathrm{n}
$$

Among that, "media" refers to a multi-media document; $k_{i}$ represents the ith key word of the document, and $\theta_{\mathrm{i}}$ stands for frequency of ith key word occurred in the multi-media document.

2)Since titles and key words of non-textual learning resources can directly reflect critical information of learning resources, the system will judge both key words in titles and key words given by resources. If the title of the multi-media document "media" contains " $m$ " key words, then $\mathrm{k}_{\mathrm{i}}(0 \leq \mathrm{i} \leq \mathrm{m})$ will be examined. If $k_{i}$ appears in the textual eigenvector model "Resource (media)", then the th-ird procedure will follow; if not, the fourth procedure will be the next one.

3)If $k_{i}$ appears in the textual eigenvector model "Resource (media)" and takes the ith place of all vectors, then make $\theta_{\mathrm{i}}=\theta_{\mathrm{i}}+3 \times_{\mathrm{c}}$. "c" stands for the proportion of times the key word occurred in tit-les of description of learning resources and the number of words in description of learning resources.

4)If $k_{i}$ doesn't occur in the textual eigenvector model "Resource (media)", the dimension number of vectors " $n=n+1$ " will be added. The updated Resource (media) will be as follows:

$$
\text { Resource }(\text { media })=\left\{\left(\mathrm{k}_{1}, \theta_{1}\right),\left(\mathrm{k}_{2}, \theta_{2}\right) \ldots\left(\mathrm{k}_{\mathrm{n}+1}, \theta_{\mathrm{n}+1}\right)\right\}
$$

Among that, $\mathrm{k}_{\mathrm{n}+1}=\mathrm{k}_{\mathrm{i}}, \theta_{\mathrm{n}+1}=3 \times_{\mathrm{c}}$. "c" represents the proportion of times the key word occurred in the title of this multi-media document "media" and the number of words of the document.

5)Normalization will go on for all key words. That is,

$$
\theta_{i}=\frac{\theta_{i} \times \log \left(\frac{M}{N_{i}}+0.01\right)}{\sqrt{\sum \theta_{i}^{2} \times \log \left(\frac{M}{N_{i}}+0.01\right)^{2}}}
$$

" $\mathrm{M}$ " refers to the total number of documents described in non-textual resources, and $\mathrm{N}_{\mathrm{i}}$ represents the number of documents which the ith key word appears. In order to avoid getting considerable eigenvector models and increasing complexity of following calculation, all non-textual learning resources are specified in descending order of their importance. And the first 20 key words will be taken as the final eigenvector models for learning resources.

\subsection{Construction of Learning Style Models of Learning Resources}

The concrete measures are: when learning resources are being uploaded, the resource style model is empty. Then, the system will recommend learning resources on the basis of learners' learning interests during initial operation. After learning these resources, learners will give a mark on the resources recommended by the system. Next, the system will pick out learners' learning style models with high satisfaction to make corresponding style models of learning resources.

In order to meet need of realization for the system, this model adopts two-dimensional vector space model in this paper. The style model consists of four dimensions: information input (visual type/verbal type), information processing (active type/passive type), content understanding (abstract type/specific type), and perception (reasonable type/intuitional type). And every dimension contains three values. For instance, the dimension of information input includes two probability values of 
visual type and verbal type, as well as its flag bit. The flag bit is used to enhance matching effect between learning style and learning resource style when personalized recommendation is undertaken. When the flag bit is " 1 ", it shows a bias to the former style type of the dimension (such as visual type); when the flag bit is “- 1 ”, it indicates a bias to the latter style type of the dimension (such as verbal type), and when the flag bit is " 0 ", it manifests the middle style type, that is, a balanced type.

The specific representation is below:

$$
\text { Rs_LS }=\{R L 1, R L 2, R L 3, R L 4\}=\left\{\left(\mathrm{P}_{11}, \mathrm{P}_{12}, \log _{1}\right)\left(\mathrm{P}_{21}, \mathrm{P}_{22}, \mathrm{Log}_{2}\right)\left(\mathrm{P}_{31}, \mathrm{P}_{32}, \mathrm{Log}_{3}\right)\left(\mathrm{P}_{41}, \mathrm{P}_{42}, \log _{4}\right)\right\}
$$

Among that, $\mathrm{P}_{\mathrm{ij}}$ (The value of “i” can be 1, 2, 3 or 4, and the value of “j” can be 1 or 2 ) refers to the respective probability values of eight types under four dimensions; $\log _{i}$ (The value of "i" can be $1,2,3$ or 4) stands for the flag bit of the ith dimension. For instance, if a learner's model is $\{(9 / 11,2 / 11,1)(1 / 11,10 / 11,-1)(6 / 11,5 / 11,0)(8 / 11,3 / 11,1)\}$, it means that the learner's learning style is visual type-passive type-balanced type of content understanding-reasonable type.

The specific procedures of making a learning resource style model are:

1) In the beginning, the style model for learning resources is empty;

2)In the process of learners' learning, the system will provide five optional buttons to learners for evaluation: quite satisfactory, satisfactory, general, dissatisfactory and quite dissatisfactory. For each document, those learners' learning style models, the evaluation of which are quite satisfactory and satisfactory, are selected to help to modify style models of learning resources. The value of every dimension can be calculated as follows:

$$
P(\text { media })=\frac{\sum_{i=1}^{m} p_{i}+\frac{1}{3} \sum_{i=1}^{n} p_{j}}{m+n}
$$

" $m$ " refers to the number of learners whose evaluation is quite satisfactory; " $n$ " stands for the number of learners whose evaluation is satisfactory; $\mathrm{P}$ (media) represents the probability value of some type in a dimension; $\mathrm{P}_{\mathrm{i}}$ indicates the probability value of the corresponding type with learning style of those learners whose evaluation is quite satisfactory, and $P_{j}$ signifies the probability value of the consistent type with learning style of learners whose evaluation is satisfactory.

3)According to the value of every dimension of the resource style model given by the second procedure, the resource style model should be steady after a period of testing.

4)Normalization will go on for $P_{i 1}$ and $P_{i 2}(1 \leq i \leq 4)$ of every dimension; the value of $\log _{i}$ of the flag bit can be determined on the basis of $\mathrm{P}_{\mathrm{i} 1}$ and $\mathrm{P}_{\mathrm{i} 2}$, and the resource style model can be finally obtained.

\section{Conclusion}

Personalized intelligent learning system has become one of study hotspots on intelligent education nowadays, and study findings about it play a vital role in personalized teaching. On the basis of learners' personalities, this system will voluntarily recommend learning resources that fill learners' practical requirements so as to help to improve learners' learning efficiency and learning quality. Since among learners' personalities, learning interest and learning style are two principal features which can affect learners' learning efficiency, this paper focuses on making resource models and style models in accordance with learning resources; then updating learning style models of learning resources on the basis of learners' learning style and their evaluation on resources recommended by the system; next, reasonably recommending learning resources to learners through scientific recommendation technology, and finally achieving personalized teaching more efficiently and realizing teaching philosophy of "teaching students in accordance of their aptitude”. 


\section{References}

[1] Koedinger, A. K. R., Brunskill, E., Baker, R. S. J. \& Mclaughlin, E. A. New Potentials for DataDriven Intelligent Tutoring System Development and Optimization. 1-17 (2011).

[2] Yu, S., Yang, X., Cheng, G. \& Wang, M. From Learning Object to Learning Cell : A Resource Organization Model for Ubiquitous Learning. 18, 206-224 (2015).

[3] Sciences, S. \& Publications, S. The Effect of Learning Styles on Education and the Teaching Process Đ brahim Ya ş ar Kazu Faculty of Technical Education, Department of Educational Sciences ,. 5, 85-94 (2009).

[4] Lu, J., Wu, D., Mao, M., Wang, W. \& Zhang, G. Recommender System Application Developments : A Survey. Decis. Support Syst. 74, 12-32 (2015).

[5] Montgomery, S. M. \& Groat, L. N. Student Learning Styles and Their-Implications for Teaching. (2015).

[6] Li, N. Approaches to learning : Literature review Na Li. Int. Baccalaureate Organ. 5-32 (2012).

[7] Hoffart, J., Suchanek, F. M., Berberich, K. \& Weikum, G. YAGO2 : A spatially and temporally enhanced knowledge base from Wikipedia. Artif. Intell. 194, 28-61 (2013).

[8] Luke, A. \& Freebody, P. Further Notes on the Four Resources Model. http://www.readingonline.org/research/lukefreebody.html 1-6 (1999).

[9] Halim, N. POLICY-BASED HIERARCHICAL MANAGEMENT OF SHARED RESOURCES IN A GRID ENVIRONMENT. United States Pat. Appl. Publ. 1, (2006).

[10] Patel, A. \& Kinshuk, K. Intelligent Tutoring Tools in a Computer-Integrated Learning Environment for Introductory Numeric Disciplines. Innov. Educ. Train. Int. 34, 200-207 (1997).

[11] Chan, N. N., Roussanaly, A. \& Boyer, A. in Open Learning and Teaching in Educational Communities: 9th European Conference on Technology Enhanced Learning, EC-TEL 2014, Graz, Austria, September 16-19, 2014, Proceedings (eds. Rensing, C., de Freitas, S., Ley, T. \& MuñozMerino, P. J.) 302-316 (Springer International Publishing, 2014). doi:10.1007/978-3-319-112008_23

[12] Moschitti, A. \& Zanzotto, F. M. Syntactic / Semantic Structures for Textual Entailment Recognition. 1020-1028 (2010).

[13] Sokoine, C. Using Mobile Phones for Teaching and Learning Purposes in Higher Learning Institutions: the Using Mobile Phones for Teaching and Learning Purposes in Higher Learning Institutions : the Case of Sokoine University of Agriculture in Tanzania. (2012).

[14] Mcilroy, I. G. T., Kees, J. E. \& Kalscheuer, J. A. HEALTH CARE MANAGEMENT SYSTEM AND COMPARING USER'PROPOSED AND FOR MANAGING MEDICAL TREATMENTS RECOMMENDED RESOURCES REQUIRED FOR TREATMENT. United States Pat. 5583758, (1996).

[15] Andre, J. \& Rogers, S. Using verbal and blind-walking distance estimates to investigate the two visual systems hypothesis. Percept. Psychophys. 68, 353-361 (2006).

[16] Chen, N. \& Hsieh, S. EFFECTS OF SHORT-TERM MEMORY AND CONTENT. Lang. Learn. Technol. 12, 93-113 (2008).

[17] Downes, S. Models for Sustainable Open Educational Resources What Resources ? Interdiscip. J. Knowl. Learn. Objects 3, (2007).

[18] Sippl, C. J. in Macmillan Dictionary of Microcomputing 350-411 (Macmillan Education UK, 1985). doi:10.1007/978-1-349-17843-8_19 
[19] Soller, A., Goodman, B., Linton, F. \& Gaimari, R. in Intelligent Tutoring Systems (eds. Goettl, B. P., Halff, H. M., Redfield, C. L. \& Shute, V. J.) 186-195 (Springer Berlin Heidelberg, 1998). doi:10.1007/3-540-68716-5_24

[20] Cha, H. J. et al. in Intelligent Tutoring Systems (eds. Ikeda, M., Ashley, K. D. \& Chan, T.-W.) 513-524 (Springer Berlin Heidelberg, 2006). doi:10.1007/11774303_51 